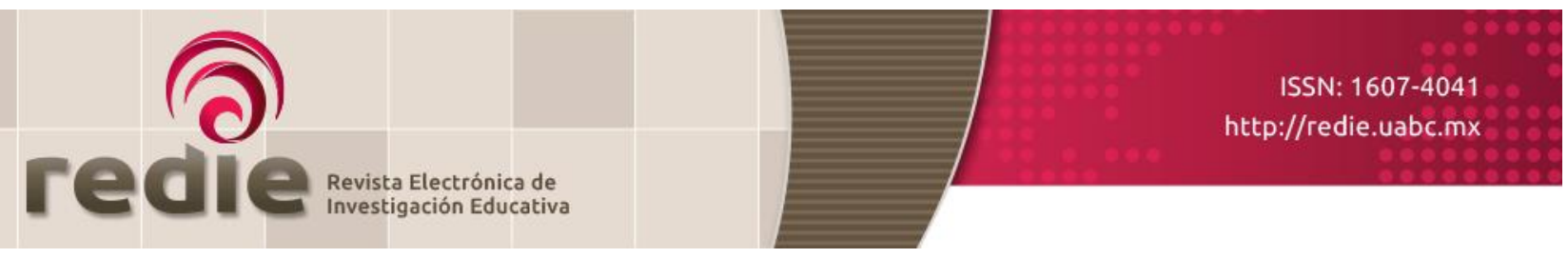

Vol. 19, Núm. 3, 2017

\title{
Gestión del conocimiento en investigadores de la Universidad de Guadalajara (México)
}

\author{
Researchers' Knowledge Management in the University \\ of Guadalajara, Mexico
}

\author{
Germán Víctor Manuel Argueta (*) german.argueta@uacj.mx \\ Carmen Patricia Jiménez (*) pjimenez@uacj.mx \\ (*) Universidad Autónoma de Ciudad Juárez \\ (Recibido: 23 de abril de 2015; Aceptado para su publicación: 26 de noviembre de 2015) \\ Cómo citar: Argueta, G. V. y Jiménez, C. P. (2017). Gestión del conocimiento en investigadores de la Universidad de Guadalajara \\ (México). Revista Electrónica de Investigación Educativa, 19(3), 1-9. https://doi.org/10.24320/redie.2017.19.3.1151
}

\begin{abstract}
Resumen
Para las universidades, el conocimiento es la materia prima y el producto en la formación de nuevos profesionistas, por lo que analizar cómo se gestiona es relevante ante el sinnúmero de estudios sobre la Gestión del Conocimiento (GC) en distintas organizaciones, no así en las instituciones de educación. El objetivo del presente estudio es analizar la GC de investigadores adscritos a cuerpos académicos del Centro Universitario de Ciencias Económico Administrativas de la Universidad de Guadalajara. El estudio es descriptivo correlacional, con enfoque cuantitativo, se aplicó un cuestionario de 30 ítems a 54 participantes. Los resultados revelan que todas las dimensiones estudiadas tienen una fuerte relación entre sí, y que el grado de consolidación de los cuerpos académicos no tiene relación significativa en ninguna de las dimensiones de la GC.
\end{abstract}

Palabras clave: Gestión del conocimiento, cuerpos académicos, universidad.

\section{Abstract}

For universities, knowledge is both the raw material and the product in training new professionals, so analyzing how it is managed is pertinent in the face of the countless studies on knowledge management (KM) in different organizations, but not in educational institutions. This study aims to analyze KM in researchers attached to academic groups in the University Center for Economic and Administrative Sciences in the University of Guadalajara (Universidad de Guadalajara). The study is descriptivecorrelational and uses a quantitative approach. A 30-item questionnaire was administered to 54 participants. The results show a strong relationship between all the dimensions studied, and that the degree of consolidation of academic groups is not significant in any of the dimensions of KM. 


\section{Introducción}

En la llamada "sociedad del conocimiento", este último reside en la mente del individuo que lo interioriza y racionaliza, al adquirirlo a través de la experiencia individual o grupal. En ese sentido, la universidad como generadora y difusora del conocimiento a través de sus docentes e investigadores, forma talento humano con capacidad crítica y reflexiva, que ayuda a las organizaciones a obtener ventajas competitivas por medio de innovaciones y resolución de problemas (Correa, Rosero y Segura, 2008; Gaviria, 2008; Magaña, Aguilar, Surdez y Quijano, 2013; Mu-Yen, Mu-Jung y Yu-Chen, 2009; Rincón, Martínez y Jiménez, 2005).

Por ello -y ante el cúmulo de conocimientos que se generan en las organizaciones- la gestión del conocimiento (Gc) se convierte en una herramienta y se define como el proceso sistemático que busca la identificación y adquisición de información para generar, capturar y organizar el conocimiento (Cordero y García, 2010; Rivas, Morales, Peña, Sotomayor y Aragón, 2002).

En las universidades, como parte del sistema educativo de México, se han creado políticas que promueven nuevas formas de estimular la generación y aplicación del conocimiento. Una de éstas es la conformación de Cuerpos Académicos (CA) dentro del Programa para el Desarrollo del Profesorado ${ }^{1}$ (PRODEP) que coordina la Secretaría de Educación Pública (SEP) (López, 2010; Mazzotti, González y Villafuerte, 2011; Nava-Rogel y Mercado-Salgado, 2011; Radi y Jomah, 2010).

El PRODEP (2016) define a los CA como "un conjunto de profesores que comparten una o más líneas de estudio, cuyos objetivos y metas están destinados a la generación y/o aplicación de nuevos conocimientos", y los clasifica en tres tipos: Formación (CAEF), En Consolidación (CAEC) y Consolidados (CAC).

El desarrollo de los CA en México, de acuerdo a López (2010), ha ido en aumento al registrar 2,971 en el año 2003; 3,402 en 2007; 3,523 en 2010 y 4,087 en 2012, siendo significativa la disminución de CAEF, dado que el primer año el $91 \%$ era CAEF, bajando a $71.16 \%, 53 \%$ y $50 \%$, respectivamente, haciendo que se incrementaran los otros niveles: los CAEC pasaron del 7\% en el 2003 al 30\% en el 2012, y el mismo efecto tuvieron los CAC, que pasaron en ese mismo lapso de un $2 \%$ a un $20 \%$.

En lo que respecta a la Universidad de Guadalajara (UDG) tenía registrados 190 CA en el año 2001, para el 2002 aumentó a 350 y disminuyó dos en el 2004; sin embargo, para el 2013 estaban registrados 374, de los cuales 199 eran CAEF, 112 CAEC y 63 CAC. El Centro Universitario de Ciencias Económico Administrativas (CUCEA) contaba al 2013 con 45 CA reconocidos: 8 consolidados, 10 en consolidación y 27 en formación (PROMEP, 2006; UDG, 2013).

Aunque el progreso de los CA ha sido sustancial en las instituciones de educación superior de México, existen pocos estudios de la GC enfocados en los investigadores que integran estos grupos universitarios, entre los que se encuentran los realizados por Magaña et al. (2013) en Tabasco; Mijangos y Manzo (2012) en el sur y sureste del país; Romo, Villalobos y Guadalupe (2012) y Topete, Bustos y Bustillos (2012) en Jalisco. Dado la escasez de estudios empíricos en dicho contexto esta investigación tiene como objetivo analizar la GC entre los investigadores adscritos a CA del CUCEA, enfocándose en las dimensiones de proceso de GC en la institución, creación del conocimiento, trabajo en equipo, transferencia del conocimiento, y tecnología y proceso de Gc.

La investigación se fundamenta en la Teoría de la Creación del Conocimiento desarrollada por Nonaka y Takeuchi (1999), conforme a autores como Donante y Guadamillas (2008); Gaviria (2008); Gaviria, Mejía y Henao (2007); Magaña et al. (2013); Mijangos y Manzo (2012); Rivas et al. (2002); Romo et al. (2012) y Topete et al. (2012), entre otros, que dan base a la GC, ya que dicha corriente se justifica en dos

\footnotetext{
${ }^{1}$ El Programa de Mejoramiento del Profesorado tuvo una modificación en el año 2014, cambiando de nombre a Programa para el Desarrollo Profesional Docente (PRODEP), pero por convenir a este estudio se utilizó la nomenclatura PROMEP (SEP, 2015).
} 
dimensiones, una epistemológica y otra ontológica; la primera se refiere al conocimiento tácito además del explícito y la segunda a que el saber parte de lo individual pasando por a lo grupal, organizacional e interorganizacional.

Esta transición del conocimiento se da en forma de espiral en un modelo integrado por cuatro etapas: socialización, se comparte el conocimiento tácito; exteriorización, es la conexión de lo tácito y lo explícito expuesta en concepto; combinación es la asociación de lo explícito a lo explicito por medio de modelos o sistemas de información; e interiorización, es la conversión de lo explícito a lo tácito creando nuevos paradigmas y modelos mentales acordes con los cambios del entorno (Nonaka y Takeuchi, 1999; Romo et al., 2012).

Considerando lo anterior, y que los trabajos de Romo et al. (2012) y Topete et al. (2012) se realizaron en el estado de Jalisco, al igual que el presente trabajo, nuestra investigación busca detallar si las estructuras institucionales, funcionales y de procesos con base en las dimensiones antes expuestas tienen una implicación en la GC entre los investigadores adscritos a CA, y si con ello se da un cambio en las transformaciones que necesita la institución, para lograr una adecuada vinculación con la sociedad y organizaciones (Gaviria, 2008).

Por otro lado, en los estudios antes mencionados los objetivos eran "describir una estrategia de GC aplicada para la formación de investigadores", así como "proponer un modelo de GC para los institutos tecnológicos, que les permita incrementar su productividad académica, buscando que sea un detonante en el desarrollo social y económico de las regiones donde se localizan", aunque ambas investigaciones se basan en la GC tienen un enfoque cualitativo, ninguna de ellas hace el análisis desde sus dimensiones ni desde las diferencias que pudiera genera en la GC el grado de consolidación del CA en que estén adscritos los investigadores. Ante esto, la hipótesis a contrastar es: "La GC focalizada en las dimensiones: proceso de GC en la institución, creación del conocimiento, trabajo en equipo, transferencia del conocimiento, tecnología y proceso de GC en CA, se efectúa mejor por los investigadores adscritos a CA consolidados".

Por otra parte, los modelos de Creación del Conocimiento -KPMG Consulting, Arthur Andersen, Knowledge Management Assessment Tool (KMAT), Diagnóstico de Lee y Furey y Cross Sectorial Learning in Virtual Enterprise (Rivas et al. 2002) - sustentan las dimensiones de este estudio; sin embargo, estos fueron realizados en empresas, no en universidades, por lo que Argueta y Jiménez (2015) diseñaron un instrumento de medición nuevo a partir de otros elaborados por Donantes y Guadamillas (2008), Magaña, et al. (2013), Moreno y Caballero (2009), Pérez, Prieto y Martín (2009), Rodríguez-Ponce, Pedraja-Rejas, Delgado y Rodríguez-Ponce (2010) y Zágarra y Bonache (2005), enfocado a investigadores con las dimensiones: Proceso de GC en la Institución (PGCI), Creación del Conocimiento (CC), Trabajo en Equipo (TE), Transferencia de Conocimiento (TC), Tecnología (T) y Proceso de GC en CA (PGCCA).

Dicho lo anterior, se plantea la hipótesis 2: "Las dimensiones Proceso de GC en la Institución, Creación del Conocimiento, Trabajo en Equipo, Transferencia del Conocimiento, Tecnología y Proceso de GC en CA como componentes de la GC entre los investigadores adscritos a CA tienen una correlación significativa positiva".

\section{Metodología}

El estudio tiene un enfoque cuantitativo de tipo descriptivo-correlacional, centrándose en la medición, representación y grado de relación entre las variables implicadas (Galeano, 2004; Salkind, 1999). La población del CUCEA de la UDG en 2013 era de 243 profesores-investigadores adscritos a 46 CA reconocidos POr PROMEP. Debido a que el levantamiento de datos se realizó en cursos de verano, el diseño muestral fue no probabilístico por conveniencia, porque la selección de los participantes se hizo por accesibilidad (Martínez, Crespo y Debón, 2006; Salkind, 1999). Los cuestionarios se aplicaron en el período junio-julio 2013, algunos personalizados y otros auto administrados, de acuerdo a la disponibilidad y preferencia del investigador, obteniendo una respuesta de 71 cuestionarios, pero eliminando 17 por estar incompletos, la muestra final fue de 54. 
Para la recolección de los datos se utilizó un cuestionario de 30 reactivos y 8 preguntas sociodemográficas elaborado por Argueta y Jiménez (2015), el cual fue adaptado al contexto de estudio en cuanto al lenguaje de los ítems (Bernal, 2006), con una escala de medida tipo Likert de seis categorías -(5) Totalmente de acuerdo, (4) Más bien de acuerdo, (3) Indiferente, (2) Más bien en desacuerdo, (1) Totalmente en desacuerdo (0) No aplica. Aunque las escalas Likert se clasifican como variables cualitativas ordinales, es común encontrar que la transforman a un nivel de medida intervalar (Santos, Vitorino y Maróco, 2013) por la tradición psicométrica (Landero y González, 2009), ya que como una variante de las escalas de clasificaciones sumadas, se supone de manera aproximada el mismo valor actitudinal (del Río, 2013), por lo que en el análisis de los datos se utilizaron estadísticos de tipo paramétrico.

\section{Resultados}

De la muestra de 54 participantes, $40.7 \%$ son mujeres y $59.3 \%$ son hombres, con un rango de edad entre 32 y 72 años; $51.9 \%$ con doctorado, 42.6\% maestría, 3.7\% posdoctorado, y 1.9\% con licenciatura. Con antigüedad entre 2 a 42 años. En cuanto al grado de consolidación de los CA, 54\% son CAEF, 33\% CAEC y 13\% CAc, teniendo como tiempo mínimo adscritos 6 meses y un máximo de 20 años.

En el análisis de consistencia interna los resultados revelan un alfa de Cronbach de .915 para todo el instrumento, siendo su dimensión más alta trabajo en equipo (.802) y la más baja creación del conocimiento (.564). Estos resultados se consideran buenos al indicar una relación existente entre los ítems y no hay reiteración (ver tabla I) (Lévy y Varela, 2003).

Tabla I. Análisis descriptivo de las dimensiones de la GC

\begin{tabular}{l|c|c|c|c}
\hline Dimensión & Media & $\begin{array}{c}\text { Desviación } \\
\text { estándar }\end{array}$ & $\begin{array}{c}\text { Alfa de } \\
\text { Cronbach }\end{array}$ & ítems \\
\hline PGCI & 3.00 & 1.17 & .709 & 3 \\
CC & 3.85 & .75 & .564 & 4 \\
TE & 3.87 & .86 & .802 & 8 \\
TC & 3.84 & .71 & .733 & 5 \\
T & 3.94 & .66 & .667 & 7 \\
PGCCA & 3.97 & .78 & .658 & 3 \\
\hline GC TOTAL & & & .915 & 30 \\
\hline
\end{tabular}

Fuente: Elaboración propia.

Los resultados descriptivos de las dimensiones de la GC muestran que los investigadores son indiferentes del proceso de GC en la institución al no sentir valorada su experiencia con un medio de la formalización y documentación de las buenas prácticas para ser transferidas a través de la institución a las nuevas generaciones de investigadores, aunque no existe un consenso. De igual forma, los participantes concuerdan con Anantatmula y Kanungo (2010), respecto a que el trabajo en equipo es importante y facilita la GC, ayudando a valorar las opiniones de los profesores investigadores que integran los diferentes CA e interactuando entre ellos, compartiendo conocimientos para generar nuevas ideas por medio de investigaciones realizadas con o sin financiamiento externo e interno al interior de la institución.

Acerca de las dimensiones de Transferencia del Conocimiento y Tecnología, los investigadores señalan una tendencia aceptable en ambas, al considerar que los conocimientos están coordinados e integrados para intercambiarlos con los demás integrantes de los CA por medio de una infraestructura tecnológica que logra un mejor flujo de información y comunicación ayudando a la consagración de los CA. Por último en relación al proceso de GC en CA es favorable al interior del grupo, al ser la dimensión mejor valorada porque buscan e identifican los huecos en las líneas de generación y aplicación del conocimiento o el surgimiento de nuevas ideas, desarrollándolas para transferir el conocimiento mediante mecanismos formales documentados o fuentes convencionales o electrónicas. 
En relación a los resultados que comprueban las hipótesis de estudio se encontró lo siguiente: Para contrastar la H1 se realizó primeramente una prueba de homogeneidad de varianzas (ver tabla II), donde se obtuvo que las dimensiones de proceso de cc en la Institución, trasferencia del conocimiento, trabajo en equipo, y proceso de GC en CA son uniformes a un nivel de significancia de .05; sin embargo, en las variables de creación del conocimiento y tecnología se encontró que no existe una igualdad, al ser menores al nivel estadístico de .05 y .01, respectivamente.

Tabla II. Prueba de homogeneidad de varianzas

\begin{tabular}{l|c|c|c|c}
\hline & $\begin{array}{c}\text { Estadístico } \\
\text { de Levene }\end{array}$ & $\mathrm{df1}$ & $\mathrm{df2}$ & sig. \\
\hline $\mathrm{PGCl}$ & 2.920 & 2 & 51 & .063 \\
$\mathrm{~T}$ & 5.984 & 2 & 51 & .005 \\
$\mathrm{TC}$ & 2.628 & 2 & 51 & .082 \\
$\mathrm{CC}$ & 3.495 & 2 & 51 & .038 \\
$\mathrm{TE}$ & .895 & 2 & 51 & .415 \\
PGCCA & 2.446 & 2 & 51 & .097 \\
\hline
\end{tabular}

A causa de lo anterior se realizó la prueba Welch, y se obtuvo que entre los participantes adscritos a CAEF y CAC existe una diferencia de medias en relación a tecnología, es decir, que para los investigadores adscritos a CAC una adecuada infraestructura tecnológica contribuirá en la formación de redes para la generación y aplicación de nuevo conocimiento con sus pares en instituciones del país o del extranjero (ver tabla III).

Tabla III. Prueba Welch de igualdad de medias

\begin{tabular}{l|c|c|c|c}
\hline & Estadístico $^{2}$ & df1 & df2 & Sig. \\
\hline PGCl & .182 & 2 & 25.846 & .835 \\
$\mathrm{~T}$ & 7.443 & 2 & 29.568 & .002 \\
$\mathrm{TC}$ & .730 & 2 & 28.083 & .491 \\
$\mathrm{CC}$ & 1.631 & 2 & 31.074 & .212 \\
$\mathrm{TE}$ & .168 & 2 & 16.422 & .847 \\
$\mathrm{PGCCA}$ & 1.848 & 2 & 16.878 & .188 \\
\hline
\end{tabular}

${ }^{\mathrm{F}} \mathrm{F}$ distribuida de forma asintónica.

Una vez realizados los análisis estadísticos que comprueban la homogeneidad de varianza, se realizó un Anova para identificar alguna relación entre la GC y el grado de consolidación de los CA (ver tabla IV), encontrando que no existe una significancia estadística al ser mayores al nivel de .05. Lo que indica que no hay una diferencia de la Gc entre los investigadores de acuerdo al grado consolidación del cA al que están adscritos. 
Tabla IV. Análisis Anova de un factor

\begin{tabular}{|c|c|c|c|c|c|c|}
\hline & & \begin{tabular}{|c|} 
Suma de \\
cuadrados
\end{tabular} & gl & $\begin{array}{c}\text { Media } \\
\text { cuadrática }\end{array}$ & $F$ & Sig. \\
\hline PGCl & $\begin{array}{l}\text { Entre grupos } \\
\text { Dentro de grupos } \\
\text { Total }\end{array}$ & $\begin{array}{r}.182 \\
71.932 \\
72.114\end{array}$ & $\begin{array}{r}2 \\
51 \\
53\end{array}$ & $\begin{array}{r}.091 \\
1.410\end{array}$ & .064 & .938 \\
\hline $\mathrm{T}$ & $\begin{array}{l}\text { Entre grupos } \\
\text { Dentro de grupos } \\
\text { Total }\end{array}$ & $\begin{array}{r}1.170 \\
22.264 \\
23.434\end{array}$ & $\begin{array}{r}2 \\
51 \\
53\end{array}$ & $\begin{array}{l}.585 \\
.437\end{array}$ & 1.340 & .271 \\
\hline TC & $\begin{array}{l}\text { Entre grupos } \\
\text { Dentro de grupos } \\
\text { Total }\end{array}$ & $\begin{array}{r}.213 \\
26.635 \\
26.847\end{array}$ & $\begin{array}{r}2 \\
51 \\
53\end{array}$ & $\begin{array}{l}.106 \\
.522\end{array}$ & .204 & .816 \\
\hline$C C$ & $\begin{array}{l}\text { Entre grupos } \\
\text { Dentro de grupos } \\
\text { Total }\end{array}$ & $\begin{array}{r}1.493 \\
28.662 \\
30.155\end{array}$ & $\begin{array}{r}2 \\
51 \\
53\end{array}$ & $\begin{array}{l}.747 \\
.562\end{array}$ & 1.329 & .274 \\
\hline TE & $\begin{array}{l}\text { Entre grupos } \\
\text { Dentro de grupos } \\
\text { Total }\end{array}$ & $\begin{array}{r}.227 \\
39.366 \\
39.593\end{array}$ & $\begin{array}{r}2 \\
51 \\
53\end{array}$ & $\begin{array}{l}.113 \\
.772\end{array}$ & .147 & .864 \\
\hline PGCCA & $\begin{array}{l}\text { Entre grupos } \\
\text { Dentro de grupos } \\
\text { Total }\end{array}$ & $\begin{array}{r}2.437 \\
29.668 \\
32.104\end{array}$ & $\begin{array}{r}2 \\
51 \\
53\end{array}$ & $\begin{array}{r}1.218 \\
.582\end{array}$ & 2.094 & .134 \\
\hline
\end{tabular}

Con base en los resultados la H1 no se acepta, puesto que el nivel de consolidación de los CA no interfiere en el desarrollo de la GC entre los investigadores, revelando que lo trascendental es el conocimiento tácito que es propiedad de los investigadores que representan el capital intelectual de las instituciones de educación; por consiguiente, para convertirlo en explícito la institución debe contar con una infraestructura tecnológica que fortalezca los procesos de Gc y facilitar el trabajo en equipo, para que ayuden en la creación y transferencia del conocimiento, lo que concuerda con la Teoría de Creación del Conocimiento de Nonaka y Takeuchi (1999).

Para responder a la $\mathrm{H} 2$ presentando evidencia empírica de la GC estructurada por las dimensiones de proceso de GC en la institución, creación del conocimiento, trabajo en equipo, transferencia de conocimiento, tecnología y proceso de GC en CA se realizó un análisis de correlación de Pearson (ver tabla V), arrojando relaciones positivas y estadísticamente significativas a un nivel de .01. La correlación más fuerte se presentó entre proceso de GC en CA y Tecnología, lo cual indica que una mejor infraestructura tecnológica ayudará en el intercambio y comunicación de información para la identificación, generación, transferencia y aplicación del conocimiento entre los investigadores .

Tabla V. Análisis de correlaciones entre las dimensiones de Gc

\begin{tabular}{l|c|c|c|c|c|c}
\hline Dimensión & \multicolumn{1}{|c|}{ PGCI } & \multicolumn{1}{c|}{ CC } & TE & TC & T & PGCCA \\
\hline PGCI & 1 & & & & & \\
CC & $.647 * *$ & 1 & & & & \\
TE & $.566 * *$ & $.680 * *$ & 1 & & & \\
TC & $.695 * *$ & $.462 * *$ & $.515 * *$ & 1 & & \\
T & $.522 * *$ & $.691 * *$ & $.588 * *$ & $.520 * *$ & 1 & \\
PGCCA & $.481 * *$ & $.672 * *$ & $.553 * *$ & $.441 * *$ & $.696 * *$ & 1 \\
\hline
\end{tabular}

** La correlación es significativa en el nivel 0.01 ( 2 colas).

Del mismo modo, otras relaciones fuertes se presentan entre creación del conocimiento con tecnología, trabajo en equipo y proceso de GC en CA, revelando que la interacción entre los miembros de los CA, fortalecido por una infraestructura tecnológica que apoye a un adecuado proceso de formalización de las actividades y los productos elaborados de investigaciones, principalmente hacia el interior del grupo académico, ayudará en la generación de nuevos conocimientos que influyan en la resolución de problemas o el cumplimiento de objetivos (ver tabla V). 
De igual forma, las relaciones entre proceso de cc en la institución con transferencia del conocimiento y creación del conocimiento son fuertes, lo que expresa que una adecuada formalización de normas y buenas prácticas a través de la institución contribuirá a que los conocimientos especializados que están integrados y fragmentados sean accesibles para ser utilizados por otros miembros, así como por la comunidad en general. Asimismo, las correlaciones más bajas se presentaron entre transferencia del conocimiento con proceso de GC en CA y creación del conocimiento, lo que indica que existe una influencia -aunque no tan fuerte-, ya que dichas actividades de formalización hacia el interior de los CA no representa que se esté compartiendo conocimiento entre los mismos miembros; y no es necesario crear nuevo conocimiento para transferirlo (ver tabla V). Por consiguiente, la H2 se acepta al hacer constar empíricamente que la relación entre las dimensiones de GC contribuyen en las líneas de generación y aplicación del conocimiento entre los investigadores adscritos a CA del CUCEA, sin importar su grado de consolidación.

\section{Conclusiones}

El estudio de la GC en investigadores adscritos a CA del CUCEA ha permitido comprobar lo expuesto en las investigaciones de Romo et al. (2012) y Mijangos y Manzo (2012), y clarificar que aunque el investigador es el actor principal en este nuevo paradigma para la consolidación de los CA, es necesario tener una infraestructura institucional y funcional, así como procesos adecuados para convertir el conocimiento tácito en explícito.

Considerando que la sociedad cambia a partir de los conocimientos adquiridos, como lo expresa Akbar (2012), y siendo la universidad reconocida como generadora y difusora de estos a través de sus investigadores, nuestro estudio ayudó a determinar que la Gc (focalizándose en las dimensiones de proceso de GC en la institución, trabajo en equipo, creación del conocimiento, tecnología, transferencia del conocimiento y proceso de GC en CA) es necesaria para hacer frente a los nuevos retos y objetivos, desarrollándolas en conjunto, ya que -como se demostró- existe una fuerte asociación entre ellas.

El trabajo en equipo facilita la integración y socialización de los miembros que componen los cA no sólo de la institución sino con los pares de IES nacionales y extranjeras, ayudando a la creación de conocimiento con nuevas ideas y resolviendo problemas que afectan no sólo a la sociedad sino a la ciencia.

Los estudios realizados por Magaña et al. (2013), Mijangos y Manzo (2012), Romo et al. (2012) y Topete et al. (2012) coinciden en que es necesaria una cultura que sea flexible para responder a estrategias de GC, aspecto no abordado en este estudio, ya que nos enfocamos en determinar si este nuevo paradigma tenía incidencia entre los investigadores de acuerdo al grado de consolidacion de su CA. Se recomienda, en futuros estudios, abordar la cultura en conjunto con los factores analizados en este trabajo, y considerar también otras áreas de investigación, no sólo las económico administrativas.

\section{Referencias}

Akbar, A. (2012). On the functions of knowledge management in Azad universities: case of Shushtar university. Interdisciplinary Journal of Contemporary Research in Business, 4(5), 691-708.

Anantatmula, V. S., y Kanungo, S. (2010). Modeling enablers for successful kM implementation. Journal of Knowledge Management, 14(1), 100-113.

Argueta, G. V. y Jiménez T., C. P. (en prensa). Gestión del conocimiento en investigadores de la Universidad Autónoma de Ciudad Juárez: creación de instrumento de medición en el contexto académico y cultural del norte de México. En C. P. Jiménez, M. Leiner y J. A. Toscano, Administración contemporánea en el Noroeste de México (pp. 35-52). México: Universidad Autónoma de Ciudad Juárez.

Bernal, C. A. (2006). Metodología de la investigación. México: Pearson. 
Cordero, A. E. y García, F. (2010). Estado de la gestión del conocimiento en dos grupos de empresas del estado Carabobo y el estado Tamaulipas. Intangible Capital, 6(2), 312-333.

Correa, G., Rosero, S. L. y Segura, H. (2008). Diseño de un modelo de gestión del conocimiento para la Escuela Iberoamericana de Bibliotecología. Revista Interamericana de Bibliotecología, 31 (1), 85-108.

Del Río, D. (2013). Diccionario-glosario de metodología de investigación social. Madrid: UNED.

Donante, M. J. y Guadamillas, F. (2008). Gestión del conocimiento organizativo, innovación tecnológica y resultados. Una investigación empírica. Investigaciones Europeas de Dirección y Economía de la Empresa, 14(2), 139-167.

Galeano, M. E. (2004). Diseño de proyectos en la investigación cualitativa. Colombia: Fondo Editorial Universidad EAFIT.

Gaviria, M. M. (2008). Gestión del conocimiento y comunidades de práctica en laboratorios de investigación del Polo Científico Grenoblés en Francia. Revista Interamericana de Bilbioteconogía, 31(2), 4578.

Gaviria, M. M., Mejía, A. M. y Henao, D. L. (2007). Gestión del conocimiento en los grupos de investigación de excelencia de la Universidad de Antioquia. Revista Interamericana de Blibliotecnología, 30(2), 137-163.

Landero, R. y González, M. T. (2006). Estadística con SPSS y metodología de la investigación. México: Trillas.

Lévy, J. P. y Varela, J. (2003). Análisis multivariable para las Ciencias Sociales. Madrid: Pearson.

López, S. (2010). Cuerpos académicos: factores de integración y producción de conocimiento. Revista de Educación Superior, 39(155), 7-26.

Magaña, D. E., Aguilar, N., Surdez, E. G. y Quijano, R. A. (2013). Gestión del conocimiento en grupos de investigación en Ciencias Sociales: caso Universidad Juárez Autónoma de Tabasco, Mexico. Administración y Finanzas, 6(5), 75-93.

Martínez, P. M., Crespo, F. y Debón, A. (2006). Métodos estadísticos en economía. España: Universidad Politécnica de Valencia.

Mazzotti, G., González, C. y Villafuerte, L. F. (2011). La universidad como desafío organizacional: cuerpos académicos, identidades y contextos colaborativos en las instituciones de educación superior en México. Administración y Organizaciones, 26, 65-79.

Mijangos, J. C. y Manzo, K. S. (2012). Gestión del conocimiento de tres cuerpos académicos consolidados del área educativa. Sinéctica, 38, 1-13. Recuperado de https://sinectica.iteso.mx/index.php/SINECTICA/article/view/94

Moreno, Z. E. y Caballero, A. E. (2009). Estado de la gestión del conocimiento en una empresa de litografía de Barquisimeto, Venezuela. Cultura, Tecnología y Patrimonio, 7, 119-150.

Mu-Yen, C., Mu-Jung, H. y Yu-Chen, C. (2009). Measuring knowledge management performance using a competitive perspective: An empirical study. Expert Systems with Applications, 36, 8449-8459.

Nava-Rogel, R. M. y Mercado-Salgado, P. (2011). Análisis de trayectoria del capital intelectual en una universidad pública mexicana. Revista Electrónica de Investigación Educativa, 13(2), 166-187. Recuperado de http://redie.uabc.mx/redie/article/view/294 
Nonaka, I. y Takeuchi, H. (1999). La organización creadora de conocimiento. Cómo las compañias japonesas crean una dinámica de la innovación. México: Oxford .

Pérez, M. P., Prieto, I. M. y Martín, C. (2009). Gestionando el conocimiento a través de la gestión de recursos humanos: analisis empírico en el sector de automocion. Revista Latinoamericana de Administración, 42, 34-56.

PRODEP. (2016). Programa para el Desarrollo Profesional Docente. Recuperado de http://dsa.sep.gob.mx/cuerposacademicos.html

PROMEP. (2006). Programa de Mejoramiento del Profesorado. Recuperado de http://promep.sep.qob.mx

Radi, M. y Jomah, F. (2010). Knowledge management architecture empirical study on the Jordanian universities. European Journal of Economics, Finance and Administrative Sciences, 20, 101-114.

Rincón, Y., Martínez, M. y Jiménez, R. (2005). Formación de profesores universitarios en el núcleo LUZCOL: articulación a los procesos de gestión del conocimiento. Revista Venezolana de Información Tecnología y Conocimiento, 1, 77-93.

Rivas, L. A., Morales, J., Peña, M. D., Sotomayor, J. I. y Aragón, M. (2002). La gestión del conocimiento en organizaciones mexicanas. Investigacion Administrativa, 91, 53-75.

Rodríguez-Ponce, E., Pedraja-Rejas, L., Delgado, M. y Rodríguez-Ponce, J. (2010). Gestión del conocimiento, liderazgo. diseño e implementación de la estrategia: un estudio empírico en pequeñas y medianas empresas. Ingeniare, 18(3), 373-382.

Romo, A. E., Villalobos, M. D. y Guadalupe, L. E. (2012). Gestión del conocimiento: estrategia para la formación de investigadores. Sinéctica, 38, 1-20. Recuperado de https://sinectica.iteso.mx/index.php/SINECTICA/article/view/95

Salkind, N. J. (1999). Metodos de Investigación. México: Pearson Educación.

Santos, M. A., Vitorino, J. M. y Maróco, J. (2013). Análisis estadístico de escalas ordinales. Aplicaciones en el Área de Salud infantil y Pediatría. Revista Enfermería Global, 30, 434-445.

Secretaría de Educación Pública. (2015). Programa para el desarrollo profesional docente para el tipo superior. Recuperado de http://dsa.sep.gob.mx/prodep.html

Topete, C., Bustos, E. y Bustillos, E. S. (2012). Gestión del conocimiento para promover la productividad académica de los institutos tecnológicos en la sociedad del conocimiento. Sinéctica,38, 1-15. Recuperado de https://sinectica.iteso.mx/index.php/SINECTICA/article/view/93

Universidad de Guadalajara (2013). Informe de actividades. Recuperado de http://www.rectoria.udg.mx/sites/default/files/IA2013-2014 estadisticalnstitucional TBP v4.pdf

Zágarra, C. y Bonache, J. (2005). Equipos de trabajo para la gestión del conocimiento: la importancia de un clima adecuado. Cuadernos de Economía y Dirección de la Empresa, 22, 27-48. 